\title{
Prestimulus Oscillatory Activity in the Alpha Band Predicts Visual Discrimination Ability
}

\author{
Hanneke van Dijk, Jan-Mathijs Schoffelen, Robert Oostenveld, and Ole Jensen \\ F. C. Donders Centre for Cognitive Neuroimaging, Radboud University Nijmegen, 6500HB Nijmegen, The Netherlands
}

\begin{abstract}
Although the resting and baseline states of the human electroencephalogram and magnetoencephalogram (MEG) are dominated by oscillations in the alpha band $(\sim 10 \mathrm{~Hz})$, the functional role of these oscillations remains unclear. In this study we used MEG to investigate how spontaneous oscillations in humans presented before visual stimuli modulate visual perception. Subjects had to report if there was a subtle difference in gray levels between two superimposed presented discs. We then compared the prestimulus brain activity for correctly (hits) versus incorrectly (misses) identified stimuli. We found that visual discrimination ability decreased with an increase in prestimulus alpha power. Given that reaction times did not vary systematically with prestimulus alpha power changes in vigilance are not likely to explain the change in discrimination ability. Source reconstruction using spatial filters allowed us to identify the brain areas accounting for this effect. The dominant sources modulating visual perception were localized around the parieto-occipital sulcus. We suggest that the parieto-occipital alpha power reflects functional inhibition imposed by higher level areas, which serves to modulate the gain of the visual stream.
\end{abstract}

Key words: oscillations; perception; resting state; EEG; electroencephalography; MEG; magnetoencephalography; rhythm

\section{Introduction}

The resting state of the human brain has recently received increasing interest in human neuroimaging research (Gusnard and Raichle, 2001). What are the electrophysiological correlates of the resting or baseline states and do they serve a functional role? The ongoing human electroencephalogram (EEG) and magnetoencephalogram (MEG) during rest are dominated by posterior $\sim 10$ $\mathrm{Hz}$ oscillations, termed the alpha, or Berger, rhythm (Berger, 1929). Although these posterior oscillations are produced by large ensembles of neurons oscillating in synchrony, their role in visual perception remains unclear. Although alpha activity used to be considered an "idling" rhythm (for review, see Pfurtscheller et al., 1996), more recent proposals suggest that it either reflects active inhibition (Klimesch et al., 2007) or plays a direct role in cortical processing (Palva and Palva, 2007).

The proposal that alpha activity reflects inhibition is based on the notion that posterior brain regions are disengaged when not functionally relevant for a given task. This is among others implied by the finding that posterior alpha power increases parametrically with working memory load during retention (Krause et al., 1996; Jensen et al., 2002; Tuladhar et al., 2007). In support of the alpha inhibition hypothesis, a visuospatial working memory experiment demonstrated that alpha power during retention increased in parieto-occipital areas ipsilateral to the hemifield

Received April 24, 2007; revised Dec. 7, 2007; accepted Dec. 28, 2007.

This work was supported in the framework of The Netherlands Organization for Scientific Research, Innovative Research Incentive Schemes, Grant 864.03.007. We thank Eric Maris for providing the software for the clusterrandomization analysis and Klaus Linkenkaer-Hansen for helpful comments on this manuscript.

Correspondence should be addressed to Ole Jensen, F. C. Donders Centre for Cognitive Neuroimaging, Radboud University Nijmegen, P.0. Box 9101, 6500HB Nijmegen, The Netherlands. E-mail: ole.jensen@fcdonders.ru.nl. DOI:10.1523/JNEUROSCI.1853-07.2008

Copyright @ 2008 Society for Neuroscience $\quad$ 0270-6474/08/281816-08\$15.00/0 where the memory item was presented compared with when the item was presented in the contralateral hemifield (Medendorp et al., 2007). Furthermore, a previous working memory study engaging either the dorsal or ventral stream, showed that alpha over dorsal areas increased when the ventral stream was engaged (Jokisch and Jensen, 2007). In addition to the inhibition hypothesis, alpha activity has been proposed to play an active role in neuronal processing (Palva and Palva, 2007). This hypothesis is among others based on findings showing phase-coupling between oscillations in the alpha- and higher-frequency bands during various cognitive tasks. Related to this idea is the notion that phase-synchronization in the alpha band between frontal and posterior regions reflects a top-down drive important for anticipation (von Stein et al., 2000).

One approach of examining the role of posterior alpha oscillations in the visual system is by investigating how alpha activity correlates with visual detection; however, the experimental data on this subject are inconclusive. In a visual detection task using EEG, Ergenoglu et al. (2004) showed that prestimulus alpha activity reduced visual detection ability, whereas Babiloni et al. (2006) found the reverse result. Beyond differences in the task, a possible explanation for the diverging results is that signal from, for example, sensorimotor and posterior areas are mixing at the sensor level because of volume conduction (Hari and Salmelin, 1997). Such mixing will confound the subsequent analysis. One step toward improving discrimination between different neuronal sources is to use MEG because this technique has a better spatial resolution than EEG (Hämäläinen et al., 1993). An additional step is to use spatial filters (e.g., beamforming) to estimate the activity from the respective sources engaged (Gross et al., 2001).

In this study, we used MEG to explore the influence of pre- 
stimulus alpha-band activity on visual perception. We presented stimuli of different contrasts at detection threshold and investigated how discrimination ability was modulated by prestimulus alpha-band power.

\section{Materials and Methods}

Subjects. Students from the local university participated in the experiment. The applied detection task was highly demanding and only 8 of 21 subjects were able to perform it in a satisfactory way (see below). The eight subjects (50\% male, age $26 \pm 3$ years) were right handed, had normal or corrected-to-normal vision, and no history of neurological disorders. Informed consent was obtained after the task was explained.

Stimuli and design. Subjects were asked to focus on a fixation cross during a baseline period of random length $(2.5-3.5 \mathrm{~s})$. The stimuli were presented for $16 \mathrm{~ms}$ and consisted of a disc $\left(2.1^{\circ}\right)$ superimposed on a larger disc $\left(3.8^{\circ}\right)$ (see Fig. 1a). Subjects were asked to push a left (right) response button when they detected a difference in gray level between the discs and a right (left) button when they did not. The response hands were counterbalanced over subjects. Immediately after the response, a uniform gray disc $\left(5^{\circ}\right)$ was presented to mask the stimuli to avoid aftereffects interfering in the next trial. If the subject did not respond within $700 \mathrm{~ms}$ the mask was presented automatically. This was done to ensure a fast response from the subjects; however, also those trials were included in the analysis. If the response was later than the mask, the 2.5-3.5 s baseline period followed the response. Visual stimuli were presented using an liquid crystal display projector (PROxtraX; Sanyo, Osaka, Japan; refresh rate, $60 \mathrm{~Hz}$ ). The subjects were trained on the task for 20 min, followed by a 20 min staircase procedure to determine the subjects' individual contrast threshold. The contrast resulting in a discrimination rate of $\sim 50 \%$ was selected to be the threshold stimulus in the recording session. In the MEG recording session, the threshold stimulus was presented in $70 \%$ of the trials. A no-contrast stimulus was presented in $26 \%$ of the trials and $4 \%$ of the trials consisted of easy contrast trials. The experimental (threshold) session took between 40 and 50 min dependent on the reaction times of the subjects. Only datasets from subjects who performed the task were analyzed. Subjects with a false alarm rate $>20 \%$ on the no-contrast stimulus were rejected because it implied that they were guessing on a lot of trials. Furthermore, to have enough trials for the analysis we required at least 130 trials for both hits as misses in the threshold contrast condition. No subjects were rejected on the basis of the MEG data. The relative high number of rejected subjects is explained by the task being highly demanding in terms of physical stamina and concentration. After the discrimination task, a short recording was performed to identify the source of the posterior alpha rhythm. Subjects were instructed to close and open their eyes (in response to one and two tones, respectively) for $6 \mathrm{~s}$ in 40 trials while MEG data were acquired. In total the recording sessions lasted $\sim 66-76 \mathrm{~min}$, self-paced breaks excluded.

Data collection and analysis. A whole-head MEG system (151 axial gradiometers, VSM/CTF Systems, Port Coquitlam, British Columbia, Canada) was used for the data acquisition. The data were low-pass filtered at $150 \mathrm{~Hz}$ and digitized at $600 \mathrm{~Hz}$. A bipolar electrooculogram (EOG) was recorded to later discard trials with eye blinks. Key presses were detected using an optical button box (Lumitouch). Before and after each recording session, the head position of the subjects was measured with respect to the MEG sensor array. This was done using coils placed on the ear canals and the bridge of the nose. Structural magnetic resonance (MR) images were acquired using a 1.5 T Sonata scanner (Siemens, Erlangen, Germany). The MEG data were aligned to the structural MR data, according to the MEG coils at the anatomical landmarks.

The Matlab (MathWorks, Natick, MA) package Fieldtrip was used for data analysis (http://www.ru.nl/fcdonders/fieldtrip). Trials contaminated by artifacts, such as eye-blinks and sensor jumps, were rejected. Only trials in which the threshold stimuli had been presented were used in the subsequent analysis.

The oscillatory activity with respect to hits and misses was characterized by calculating the power in the $1 \mathrm{~s}$ interval preceding the stimulus. A multitaper spectral estimation method was applied (Percival and Wal-
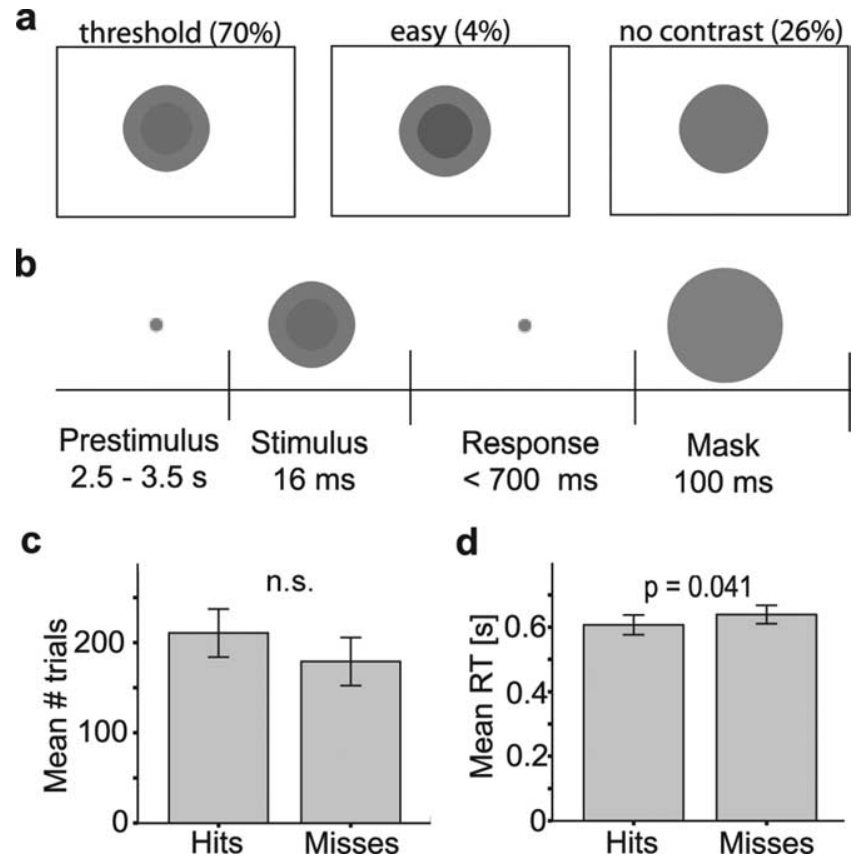

Figure 1. Task used to determine visual discrimination ability and behavioral data. $\boldsymbol{a}$, The stimuli consisted of a smaller disc superimposed on a larger disc with different contrasts (gray levels). Contrasts resulting in $\sim 50 \%$ detection defined the threshold stimulus. The contrasts are exaggerated here to make them clearly visible. In $70 \%$ of the trials the threshold stimulus was presented. Of the remaining trials, $4 \%$ contained an easy contrast stimulus and $26 \%$ a no-contrast stimulus. $\boldsymbol{b}$, After a fixation period with a random duration $(2.0-3.5 \mathrm{~s})$ the stimulus was presented for 16 milliseconds. In a forced-choice task, subjects reported whether they detected a contrast difference within a $700 \mathrm{~ms}$ response interval. Immediately after the response, a mask was presented. $c$, There was no significant difference between the number of trials with hits and misses. $\boldsymbol{d}$, The reaction time for hits was faster than for misses ( $p=0.041)$.

den, 1993). The data were multiplied with a set of three orthogonal Slepian tapers. Each tapered data epoch was subsequently Fourier transformed and the power-spectral densities were averaged. This procedure resulted in single-trial estimates of oscillatory power with $\pm 2 \mathrm{~Hz}$ frequency smoothing.

When analyzing the topography of the power spectra at the sensor level we computed the planar gradiometer representation of the data (Bastiaansen and Knosche, 2000). The calculated planar field gradient approximates the signals measured by physical planar gradiometers [e.g., as in Elekta (Stockholm, Sweden) Neuromag systems]. This is often advantageous when analyzing MEG signals, because the strongest field of the planar gradient signal usually is situated above the neural sources (Ahonen et al., 1993). The horizontal and vertical components of the planar gradients were estimated at each sensor location using the fields from the sensor and its neighboring sensors. The power values for the horizontal and vertical components after the spectral analysis were summed for each sensor location.

Changes in power were statistically assessed using a nonparametric randomization method identifying clusters of sensors with significant changes. This effectively corrects for multiple comparisons over sensors, in within subject comparisons (Nichols and Holmes, 2002; Maris and Oostenveld, 2007). Clusters are defined as spatially contiguous sensors where the $t$ statistics exceed a previous threshold $(p<0.05)$ with respect to two conditions. Note that the comparison based on $t$ statistics was used to identify sensors with effects exceeding a threshold for the subsequent cluster analysis; thus, the power values to be tested were not required to be normally distributed. The cluster-level test statistic was defined as the sum of the $t$ statistics of the sensors in a cluster. In a nonparametric statistical test, the type-I error rate for the complete set of 151 sensors was controlled by evaluating the cluster-level test statistic under the randomization null distribution of the maximum cluster-level test statistic. This was obtained by randomly permuting the data between the two experi- 
mental conditions within every participant. By creating a reference distribution from 1000 random sets of permutations, the $p$ value was estimated as the proportion of the elements in the randomization null distribution exceeding the observed maximum cluster-level test statistic.

Dynamic Imaging of Coherent Sources (DICS), a frequency-domain adaptive spatial filtering algorithm was applied to identify the neuronal source of interest. DICS has proven to be particularly useful when localizing oscillatory sources (Liljeström et al., 2005). The brain volume was discretized to a three-dimensional grid (5 mm spacing), and at each grid point spatial filters were constructed from the crossspectral density matrix of the MEG signals and the respective lead field. The multitaper method described above was used to estimate the Fourier-transformed data and the crossspectral density matrix. The lead fields were calculated from a multiple spheres head model (Huang and Mosher, 1997). The resulting spatial filters were applied to the power of the Fourier-transformed data for the frequency of interest (i.e., alpha) to optimally pass information of the location of interest while attenuating the activity from other regions. To reduce biases toward the center of the head, we computed the neural activity index (NAI) by dividing the estimated power at each grid point by an estimate of the noise. This noise bias is affected by the amount of data in the sense that more data results in a reduction of variance. Thus, the noise biases had to be estimated from the experimental data subject by subject. This was done by applying the spatial filter to an identity matrix scaled with the smallest singular value of the cross-spectral density matrix (Van Veen et al., 1997). To prevent this bias, we equalized the number of trials in the hits and misses conditions. For each subject we randomly selected trials from the condition with the most trials until the amount of trials was equal to the number of trials in the condition with the least trials. The resulting distribution of the NAI in the alpha band was subsequently coregistered on the subject's structural MRI. To compute the grand average of the data, the individual functional volumes were spatially normalized to a canonical brain, and subsequently smoothed with a Gaussian kernel (full width at half-maximum, $10 \mathrm{~mm}$ ), using SPM2 (http://www.fil.ion.ucl.ac.uk/spm/software/spm2) (Supplemental figure, available at www.jneurosci.org as supplemental material). Subsequently, the spatially normalized source estimates were averaged.

For the experimental conditions, the spatial filters were also used to derive estimates of the source power on a trial by trial basis ("virtual sensors"). To this end, the spatial filter at the regions of interest was applied to the Fourier transforms of single tapered data segments. The regions of interest were chosen. The contribution of the dipole at the locations of interest was estimated by performing a singular-value decomposition on the real part of the cross-spectral density matrix of the virtual sensors. Single-trial power estimates were obtained by rotating the spatially filtered Fourier transforms according to the first singular vector and subsequently averaging the power of the data segments across the tapers.

We investigated poststimulus activity by calculating the event-related fields (ERFs). Trials were low-pass filtered off-line at $30 \mathrm{~Hz}$ and baseline corrected $(-100-0 \mathrm{~ms})$. The trials were divided into hits and misses. For each subject the difference between the two conditions was calculated in the axial gradient representation after which the planar gradient representation was computed as described above. For each sensor location we
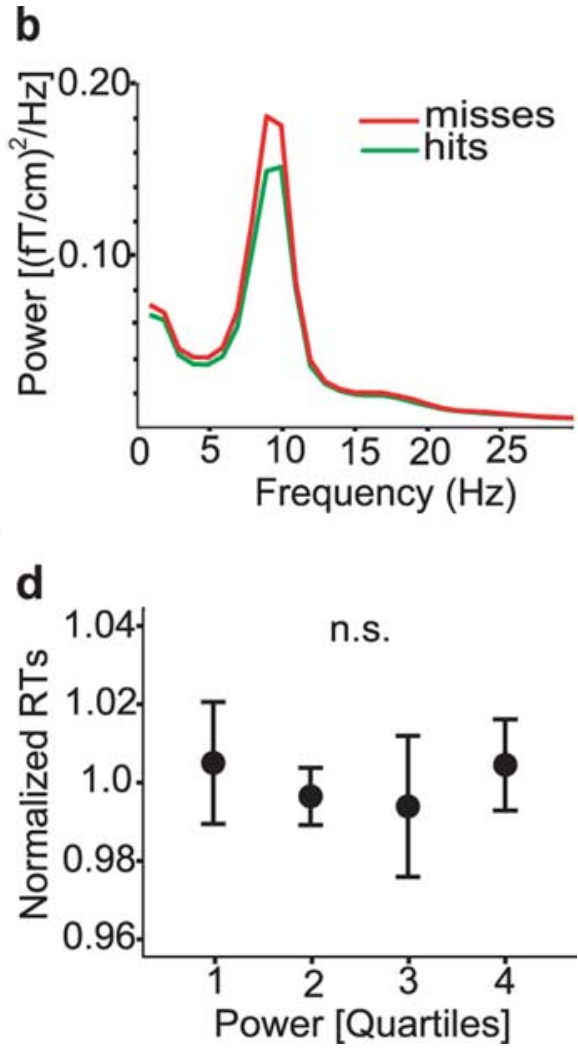

Figure 2. Characterization of visual discrimination ability in relation to the prestimulus MEG data. $\boldsymbol{a}$, Topography of the 8-12 $\mathrm{Hz}$ power of the difference between misses and hits (planar gradient) averaged over subjects. Sensors showing significantly 列 than in the fourth quartile $(p=0.018)$. $\boldsymbol{d}$, The reaction times for each quartile normalized and averaged over

then calculated the root mean square for the two planar gradient directions. Subsequently, the ERF differences were averaged over subjects.

\section{Results}

We used a task in which subjects had to report whether they could distinguish the contrast between two superimposed gray discs (Fig. 1a). First, we identified the discrimination threshold in each subject using a staircase procedure (see Materials and Methods). Subsequently, while the MEG was recorded, we presented the stimuli at discrimination threshold (70\%) randomly intermixed with catch trials.

Data were collected for 21 subjects and analyzed for the eight subjects who were able to perform the task well (see Materials and Methods). In the analysis we categorized the trials with stimuli presented at threshold into hits (detected) and misses (not detected). There was no significant differences between the number of hits and misses ( $p=0.25$, two-tailed paired $t$ test) (Fig. $1 c$ ). Reaction times were slightly faster for hits than misses $(p=$ 0.041, two-tailed paired $t$ test) (Fig. 1d).

As the first step in the MEG data analysis, we characterized the alpha power with respect to behavioral responses. The power spectra were calculated for all sensors (after conversion to the planar gradient; see Materials and Methods) in a $1 \mathrm{~s}$ prestimulus interval for hits and misses separately. Figure $2 a$ shows the alpha power $(8-12 \mathrm{~Hz})$ topography for the difference between misses 
(a) Hits and Misses combined
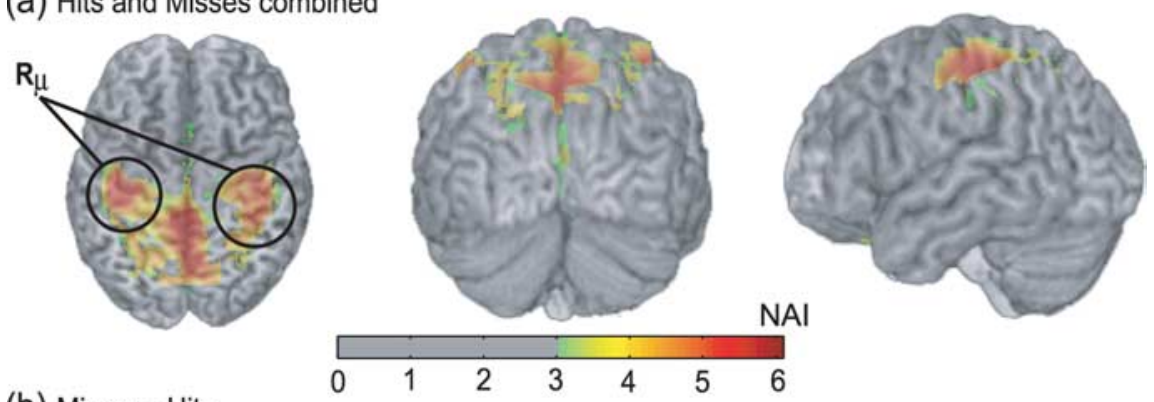

(b) Misses - Hits
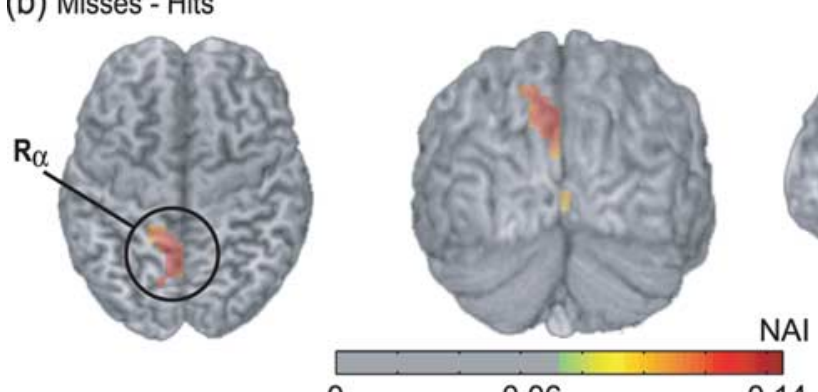$$
0
$$

0.06

0.14

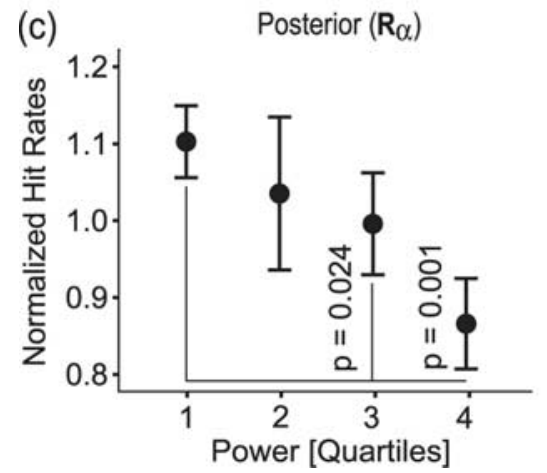

(d)

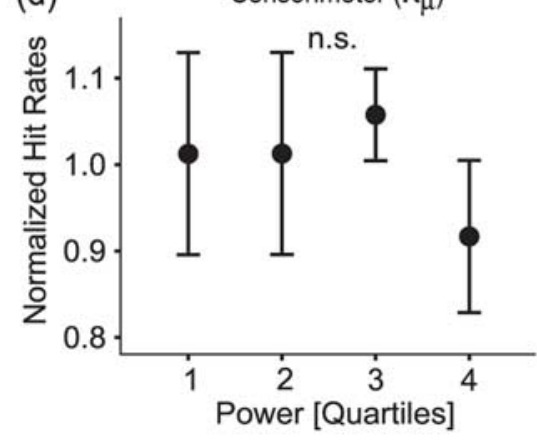

Figure 3. Source estimation of the prestimulus alpha activity and characterization of the data using virtual sensors. $\boldsymbol{a}$, The source estimates of the prestimulus $8-12 \mathrm{~Hz}$ activity for hits and misses were combined for each subject, normalized to a standard brain and then averaged. The sensorimotor mu sources are indicated $\left(R_{\mu}\right) \cdot \boldsymbol{b}$, The estimated source activity for hits subtracted from the misses. The posterior alpha source is indicated $\left(R_{\alpha}\right)$. Sources maps were thresholded with respect to $50 \%$ of the maximum. The colors represent NAls. $c$, Hit rates for the posterior alpha source $\left(R_{\alpha}\right)$. The hit rates for the third and fourth quartile differ from the first quartile ( $p=0.024$ and $p=0.001$, respectively). $\boldsymbol{d}$, Hit rates were not correlated with by the $8-12$ $\mathrm{Hz}$ activity from for the bilateral mu sources $\left(R_{\mu}\right)$. Error bars represent SEM.

and hits. The prestimulus alpha power was significantly higher for misses than hits (corrected for multiple comparisons by a cluster analysis; $p=0.008$ ). Figure $2 b$ shows the power spectra averaged over the posterior sensors belonging to the cluster with a significant effect. Note that a modulation of power $<30 \mathrm{~Hz}$ with respect to hits and misses was constrained to the alpha band. This was the case when considering the other sensors as well.

To investigate how detection ability was related to alpha power, we selected the sensors that showed a significant difference in prestimulus alpha power between hits and misses (Fig. $2 a)$. Subsequently, we estimated the average single-trial alpha power from the prestimulus intervals of these sensors for each subject. The trials were sorted according to power and divided into quartiles. Hit rates (number of hits divided by misses) were calculated for each quartile and normalized in each subject to the individual mean hit rate over all quartiles (Linkenkaer-Hansen et al., 2004) (Fig. 2c). The normalized hit rates in the first quartile were significantly larger than hit rates in the fourth quartile ( $p=$ 0.018 , repeated-measures ANOVA, simple first contrast). Hit rates for intermediate compared with low alpha power did not differ significantly (Fig. 2c). Because Linkenkaer-Hansen et al. (2004) found a quadratic relationship between somatosensory mu power and somatosensory detection rates, we investigated if there was a quadratic relationship between alpha power and visual hit rates in other sensors. We subjected the hit rates in the four bins to the following expression: hit rate (alphabin) $=a$ alpha-bin ${ }^{2}+b$ alpha-bin $+c$, where alpha-bin refers to the four alphabins (represented in the equation as -3 , $-1,1$, and 3 ). We did this for each sensor and then tested if the quadratic, $(a)$ and the linear term $(b)$ were different from 0 using the cluster randomization procedure. Only the linear term was different from 0 in posterior sensors overlapping with those in Figure $2 a$. In conclusion, we found no evidence suggesting a quadratic relationship between hit rate and alpha power. Figure $2 d$ shows the relationship between reaction times and alpha power. Reaction times were computed for each quartile and normalized according to the mean in each subject. The reaction times were not significantly related to the alpha power with respect to the four bins (repeated-measures ANOVA). Using the same analysis as for the hit rates, we found no evidence for a quadratic relationship between reaction time and alpha power.

To identify the sources producing the oscillatory activity modulating the visual detection ability, we applied a beamforming technique. First, we estimated the sources of the $1 \mathrm{~s}$ prestimulus activity by computing the NAI (Van Veen et al., 1997) in the $8-12 \mathrm{~Hz}$ band for both hits and misses (Fig. 3a). The source representations were coregistered onto the individual subjects' structural MR images, normalized to a standard brain, and averaged. The strongest source was identified in precuneus [Brodmann area (BA) 7] bordering the parieto-occipital sulcus. Additionally, we identified two bilateral sources around the sensorimotor hand areas of the central sulcus (BAs 3 and 4). These reflect the $\sim 10 \mathrm{~Hz}$ component of the sensorimotor mu rhythm (Hari and Salmelin, 1997). To identify the sources accounting for the modulation in detection ability, we subtracted the NAI for hits and misses. The source representing the difference in alpha power was localized around the parieto-occipital sulcus (Fig. 3b). This source location is consistent with the alpha power differences observed at the sensor level topographic maps (Fig. 2a). There was a tendency toward a left lateralization. However, this was mainly dominated by 4 subjects. Note that no sources accounting for the difference between the two conditions were found in sensorimotor regions. In short, sources for the $\sim 10 \mathrm{~Hz}$ prestimulus activities were found in posterior and sensorimotor regions; however, sources correlating with discrimination ability were only found in posterior regions.

To investigate hit rates as a function of $\sim 10 \mathrm{~Hz}$ activity at the sources level, we used the coordinates of the sources (one poste- 
rior alpha and two central mu sources) identified in each subject to create a "virtual sensor" by means of spatial filtering. The signals from the virtual sensors were analyzed in the same way as the sensor data. We found that the prestimulus alpha power with respect to a virtual sensor in the parieto-occipital sulcus (Fig. $3 b, R_{\alpha}$ ) strongly correlated with visual discrimination ability: the normalized hit rates decrease with an increase in alpha power (Fig. 3c) (main effect, $p=0.015$, repeatedmeasures ANOVA). The hit rate in the first quartile (representing low alpha power) was significantly higher than the hit rates in the third and fourth quartiles ( $p=$ 0.024 and $p=0.001$, respectively, repeated-measures ANOVA, simple first contrast). The effect observed for the virtual posterior sensors showed a more systematic decrease in hit rate with alpha power compared with the effect at the sensors (compare Figs. $2 c, 3 c$ ). The prestimulus power detected by the virtual sensors in the left and right sensorimotor regions (Fig. $3 b)\left(R_{\mu}\right.$ averaged over both hemispheres) did not influence the hit rates (Fig. $3 d$ ) (averaged over seven subjects because one subject was excluded because of the absence of mu sources).

Because posterior alpha power is known to increase when eyes are closed compared with when eyes are open (the classical Berger effect), this raises the question whether the posterior source we have identified corresponds to the source accounting for Berger's effect (Berger, 1929). To investigate this, we acquired MEG data when the subjects were performing a simple eyes open/closed task ( 40 epochs of 6 s of eyes opened followed by $6 \mathrm{~s}$ of eyes closed). The power spectra showed a strong alpha modulation at $8-12 \mathrm{~Hz}$ when comparing eyes closed to eyes open and it was significant in most sensors (Fig. 4a,b). As expected, the alpha activity was strongest in the posterior sensors. The topography of the difference between misses and hits does not seem to differ significantly from the Berger effect (compare Figs. 2a, 4a); however, a source analysis did reveal a difference. The location of the source accounting for the difference in $\sim 10 \mathrm{~Hz}$ activity when comparing eyes open to closed was identified to the cuneus (BA $17 / 18$ ) just posterior to the parieto-occipital sulcus. This location is more posterior compared with the source accounting for the difference between misses and hits.

To investigate whether there were systematic changes in behavior and/or alpha power modulation over the course of the experiment, we divided the trials into quartiles according to their order in the experiment. Hit rates (hits/misses) were calculated for each quartile and normalized in each subject to the individual mean hit rate over all quartiles. The normalized grand average of alpha power did not change significantly during the course of the experiment $(p=0.17$ ) (Fig. $5 a)$. Reaction times did not differ significantly between quartiles either ( $p=0.27$ ) (Fig. $5 b$ ). With respect to hit rates, we observed a systematic decrease in performance from the first compared with the rest of the quartiles ( $p=$ $0.041, p=0.01$, and $p=0.008$ respectively, repeated-measures b

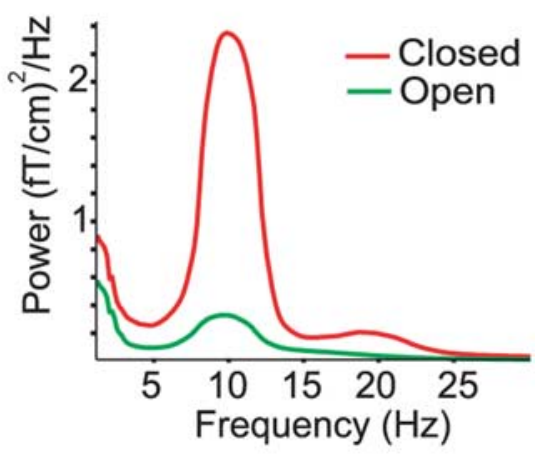

0

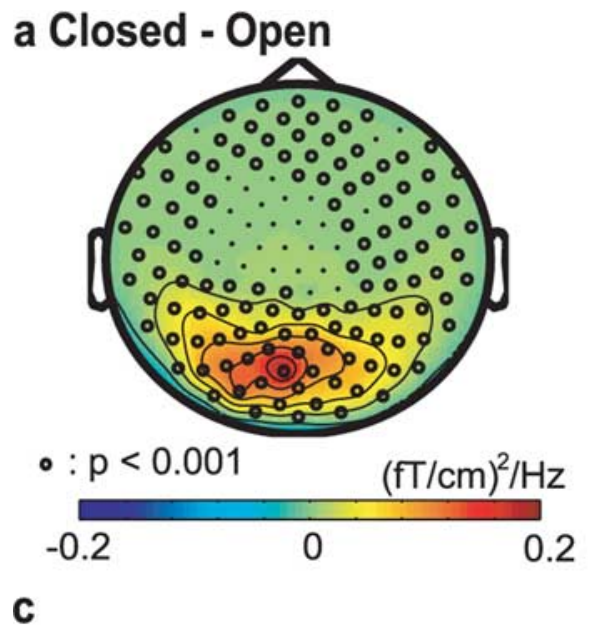

0.2

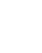

Figure 4. Characterization of the 8 $-12 \mathrm{~Hz}$ alpha activity with respect to the eyes open/closed task. $\boldsymbol{a}$, Topography of the $8-12$ activity for eyes closed and open were subtracted for each subject, normalized to a standard brain and then averaged. The source maps were masked with respect to $50 \%$ of the maximum. The colors represent NAls.

ANOVA, simple first contrast) (Fig. $5 c$ ). We conclude that, although hit rates did decrease over the course of the experiment, this decrease was not associated with significant changes in reaction times or alpha power.

To investigate brain activity reflecting hits versus misses after stimulus presentation, we calculated the combined planar gradient of the ERFs. As seen in Figure 6 we observed a reliable difference in the early ERFs 90-135 ms after stimulus presentation. The ERF for hits was bigger then the ERF for misses. The topography reflecting the differences was clearly posterior and somewhat left lateralized. The topographies with respect to ERF differences and prestimulus alpha differences (Fig. 2a) had quite a similar distribution. Thus, on the basis of these data, it is likely that the same brain regions are responsible for producing the differences in ERFs and alpha power. Source modeling would have helped to clarify this issue; however, because of the weak ERFs, we were not able to reliably construct a source model accounting for the difference between hits and misses. On this basis, we tentatively conclude that the alpha activity does modulate the excitability of posterior visual areas. These modulations in excitability are expressed in the magnitude of the ERFs reflecting visual perception in the same regions.

\section{Discussion}

We have demonstrated that prestimulus alpha power in humans correlates with contrast-discrimination ability in a simple visual task. An increase in posterior alpha power correlated with a decrease in discrimination ability. Source analysis revealed that 
a

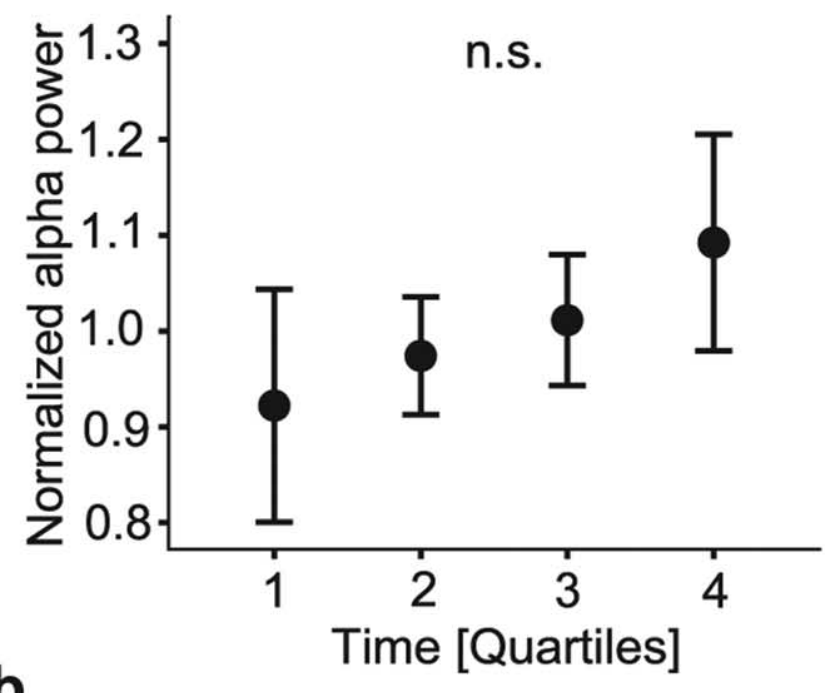

b
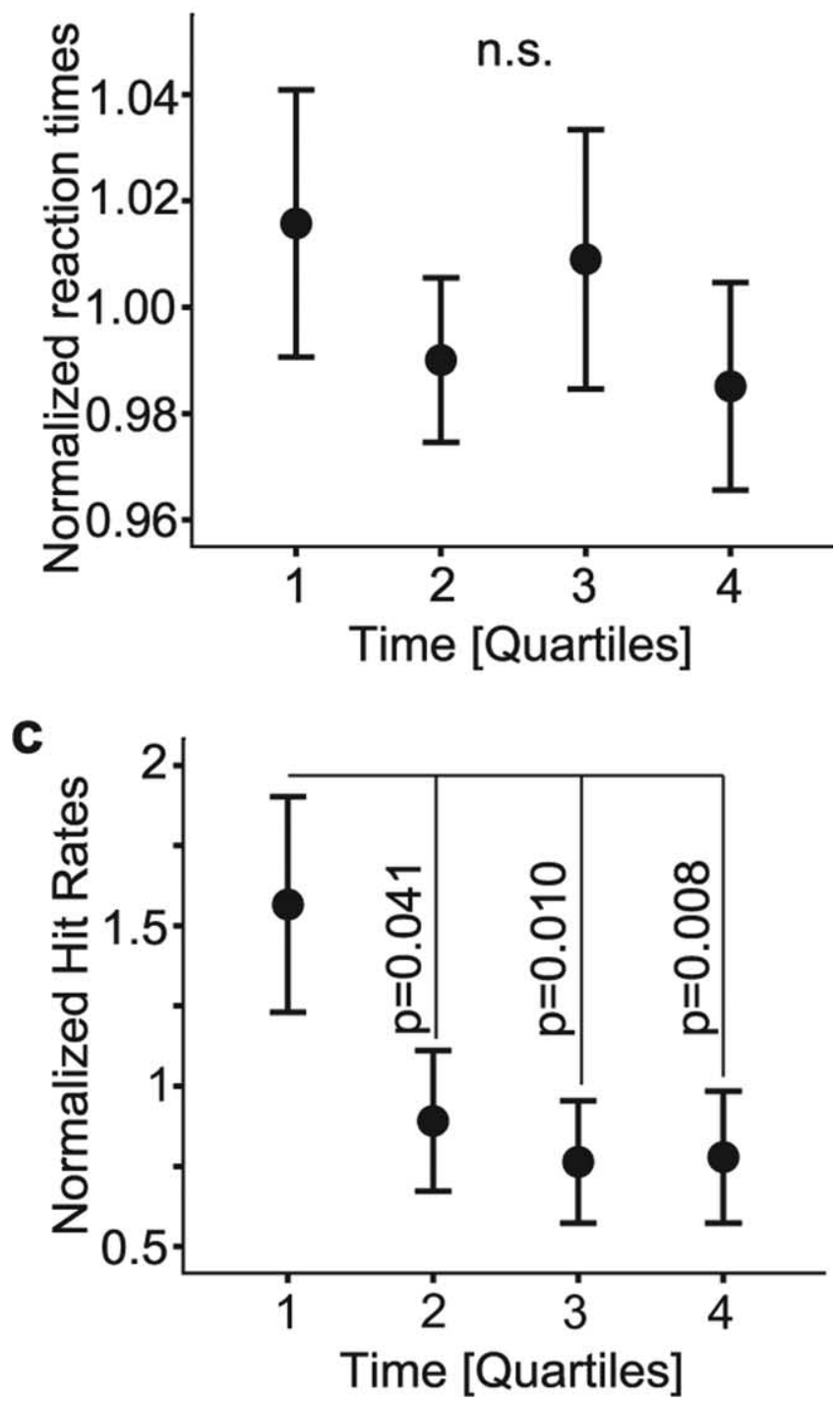

Figure 5. Alpha power and reaction times during the experiment. $\boldsymbol{a}, \boldsymbol{b}$, The alpha power $(\boldsymbol{a})$ and reaction times $(\boldsymbol{b})$ did not change during the course of the experiment. $\boldsymbol{c}$, Hit rates were significantly stronger in the first quartile compared with the rest. Error bars represent SEM. $\sim 10 \mathrm{~Hz}$ activity around the parieto-occipital sulcus was accompanied with a modulation in discrimination ability. Although reaction times were faster for hits than for misses, they did not vary systematically with alpha power. Thus, we suggest that the changes in visual discrimination ability are modulated by oscillatory activity in the alpha band, which serves to reduce the gain of the visual dorsal stream.

Our findings are consistent with the hypothesis that increases in posterior alpha activity reflect inhibition or disengagement of posterior areas (Ray and Cole, 1985a,b; Vanni et al., 1997; Klimesch et al., 2000; Jensen et al., 2002; Cooper et al., 2003; Tuladhar et al., 2007). One functional interpretation of the inhibition hypothesis is that the gain of the dorsal visual stream is reduced with alpha power (Jokisch and Jensen, 2007). The gain would serve to "gate" the information passed from occipital to dorsal parietal areas. The gain would be inversely related to alpha activity in the parieto-occipital sulcus. We suggest that a reduced gain is reflected in the visual evoked fields produced in posterior areas (Fig. 6). As a consequence, subtle details are less likely to elicit a conscious percept. Although alpha activity is likely to be controlled by a top-down mechanism such as attention (Worden et al., 2000; Medendorp et al., 2007), it is at this stage not clear which frontal areas exert this control. It should be mentioned that our findings pertain to how alpha power modulates visual detection ability. A previous study by Palva and Palva (2007) argues that alpha activity could play an active role in cognitive processing. This argument is based on findings demonstrating cognitive modulation of the phase relationship between alpha sources rather than modulations in power.

What is the functional benefit of inhibiting posterior areas? One advantage might be to reduce the flow of visual information, which could interfere with processing in higher level areas such as working memory maintenance This hypothesis is among others based on findings demonstrating that alpha power increases during working memory retention (Jensen et al., 2002; Tuladhar et al., 2007). Consistent with this notion it was demonstrated in visual cued attention experiments that alpha power increased in occipital areas ipsilateral to the hemifield attended to, compared with when this hemifield was ignored (Worden et al., 2000; Fu et al., 2001; Yamagishi et al., 2005; Kelly et al., 2006; Thut et al., 2006).

We found that the alpha source representing the difference between misses and hits is anterior to the source of the "classical" alpha activity identified in the eyes open/closed condition (compare Figs. $3 b, 4 c$ ). Our results indicate that the alpha sources anterior and posterior to the parieto-occipital sulcus serve different functions. Given the spatial resolution of MEG, we cannot tell whether this reflects two distinct sources or one larger source differentially modulated over the dorsal axes. Given that several studies have argued for a top-down-driven attention system engaging the dorsal stream, the modulation of occipital and parietal areas is likely to be related (Corbetta and Shulman, 2002; Fox et al., 2006). More studies are required to establish if alpha sources anterior and posterior to the parieto-occipital sulcus have different functional roles.

One concern is that the correlation between alpha power and discrimination ability is primarily explained by changes in vigilance. This concern is based on the observation that both reaction times and alpha power had higher values for misses than hits (Fig. 1d). However, when reaction times were correlated with alpha power (Figs. $2 d, 3 d$ ), it was clear that no correlation existed. Additionally, although hit rates did decrease over the course of the experiment, this was not associated with systematic changes in alpha power or reaction times (Fig. 5). Based on these findings, we conclude that the 
changes in discrimination ability with alpha activity are not explained by changes in vigilance.

Previous studies on prestimulus alpha activity and detection ability have yielded inconsistent results (Babiloni et al., 2003; Ergenoglu et al., 2004). One explanation for the discrepancies might stem from the fact that several sources in the human brain produce oscillatory activity in the alpha band (Hari and Salmelin, 1997). This in particular becomes a problem when analyzing EEG and MEG data because the activity from different sources will mix at the sensor level because of volume conduction and cross talk. By analyzing the signals at the source level using spatial filters, we were able to disentangle the different $\sim 10 \mathrm{~Hz}$ sources resulting in an improved correlation between alpha power and detection ability (Fig. 3, compare $a, b$ ). In conclusion, the combination of MEG with spatial filters allowed us to robustly demonstrate that only posterior alpha power with sources around the parieto-occipital sulcus predicts discrimination ability.

Although the $\sim 10 \mathrm{~Hz}$ sensorimotor mu rhythm did not predict visual discrimination ability, this rhythm has been shown to correlate with somatosensory detection in an MEG study by Linkenkaer-Hansen et al. (2004). In sensors over sensorimotor areas, they showed that intermediate prestimulus $\sim 10 \mathrm{~Hz}$ power was associated with the highest somatosensory detection ability. One possibility is that the $\sim 10 \mathrm{~Hz}$ sensorimotor mu rhythm plays a different role for somatosensory processing than the posterior alpha rhythm plays for visual processing; however, a direct comparison is complicated by the finding that the strongest alpha sources modulating the detection ability were around the parieto-occipital sulcus rather than primary visual cortex. In addition, Linkenkaer-Hansen et al. (2004) found that an increase in alpha power in posterior sensors correlated with an increase in somatosensory detection ability. This result is consistent with the inhibition hypothesis of posterior alpha activity: when a subject is engaged in somatosensory detection, inhibiting the visual stream might serve to allocate resources to the sensorimotor system.

The influence of the ongoing state of the brain on task-related activity has been the focus of recent studies applying functional magnetic resonance imaging (fMRI). An fMRI study showed that the blood oxygen level-dependent (BOLD) response in early visual areas correlated positively with performance in a visual pattern detection task (Ress et al., 2000). The authors infer that the increase in BOLD correlating with detection ability reflects a change in local cerebral blood flow because of an attentionrelated increase in prestimulus firing rates of a large population of neurons in visual cortex. Although the poor temporal resolution of fMRI makes it difficult to interpret the reported BOLD increase as prestimulus activity, we would like to extend the hypothesis. Because combined fMRI and EEG studies have demonstrated a negative correlation between alpha power and the BOLD signal (Goldman et al., 2002; Laufs et al., 2003), the increase in the visual cortex BOLD signal correlating with visual detection ability could be explained by a decrease in prestimulus alpha power. More research possibly combining EEG and $\mathrm{AMRI}$ is required to further investigate this hypothesis.

Our findings suggest that prestimulus alpha power reflects functional inhibition of the visual pathway. What are the neuro- b

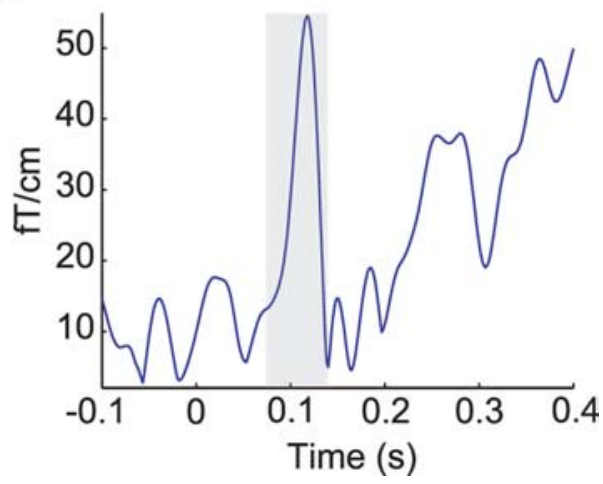
tion with an increase in alpha activity? Jones et al. (2000) proposed a computational model involving mutually connected excitatory and inhibitory neurons. The model works as follows: during the alpha cycle, a group of inhibitory neurons fires. The firing results in GABAergic inhibition of the excitatory neurons. These neurons are initially hyperpolarized by the GABAergic inhibition, but because of rebound excitation they well eventually fire. This excitatory firing in return activates the inhibitory neurons and a new cycle starts. When considering realistic kinetics and time scales of the receptors and synapses involved, this mechanism will produce $\sim 100 \mathrm{~ms}$ rhythmic cycles. The periods of hyperpolarization increase with GABAergic inhibition, as does the rebound excitation. According to this scheme, the increase in functional inhibition with alpha activity might be explained by the GABAergic feedback silencing the excitatory cells involved in conveying visual information (Jones et al., 2000). It should be mentioned that models have been proposed in which GABAergic feedback is important for the generation of gamma and beta oscillations (Traub et al., 1999; Jensen et al., 2005). In these models it is the direct inhibitory feedback to excitatory and inhibitory neurons allowing for the fast oscillations to emerge. This is in contrast to the model of Jones et al. (2000), where the rhythm generation is a consequence of rebound excitation after a GABAergic inhibition. This model could be extended to include thalamic interactions, which are known to play a role in the generation of the alpha rhythm as well (Lopes da Silva, 1991; Rougeul-Buser and Buser, 1997; Hughes and Crunelli, 2005). Complementary to this idea is that the gain of the visual stream is modulated by the alpha rhythm. Previous experimental and theoretical work have investigated the gain properties of neocortical neurons from sensory areas (Chance et al., 2002). It was demonstrated that the gain of these neurons was modulated by background synaptic input. Although the synaptic input was assumed to be a consequence of random background firing with no temporal structure, this framework might also be applied to understand gain modulation in the presence of rhythmic background activity. The rhythmic background activity is likely to be a consequence of a top-down drive, which determines the gain of the visual system (i.e., there is a reduction in gain when alpha activity increases). It has been proposed that gain modulation is a general computational principle of the CNS (Salinas and Thier, 2000). We propose the working hypothesis that gain modulation in general is achieved by changing the oscillatory properties in the alpha band in relevant networks.

In conclusion, the ongoing state of the brain characterized by 
spontaneous alpha activity modulates task-related behavior. Therefore, this activity should not be disregarded when performing electrophysiological and functional imaging studies. Moreover, it may be beneficial to monitor the alpha activity not only in subjects performing a task in an experimental setting, but also in people performing real-life jobs where high visual performance is required.

\section{References}

Ahonen AI, Hamalainen MS, Ilmoniemi RJ, Kajola MJ, Knuutila JE, Simola JT, Vilkman VA (1993) Sampling theory for neuromagnetic detector arrays. IEEE Trans Biomed Eng 40:859-869.

Babiloni C, Brancucci A, Babiloni F, Capotosto P, Carducci F, Cincotti F, ArendtNielsen L, Chen AC, Rossini PM (2003) Anticipatory cortical responses during the expectancy of a predictable painful stimulation. A high-resolution electroencephalography study. Eur J Neurosci 18:1692-1700.

Babiloni C, Vecchio F, Bultrini A, Luca Romani G, Rossini PM (2006) Preand poststimulus alpha rhythms are related to conscious visual perception: a high-resolution EEG study. Cereb Cortex 16:1690-1700.

Bastiaansen MC, Knosche TR (2000) Tangential derivative mapping of axial MEG applied to event-related desynchronization research. Clin Neurophysiol 111:1300-1305.

Berger H (1929) Über das elektroenkephalogramm des menschen. Arch Psychiatr Nervenkr 87:527-570.

Chance FS, Abbott LF, Reyes AD (2002) Gain modulation from background synaptic input. Neuron 35:773-782.

Cooper NR, Croft RJ, Dominey SJ, Burgess AP, Gruzelier JH (2003) Paradox lost? Exploring the role of alpha oscillations during externally vs. internally directed attention and the implications for idling and inhibition hypotheses. Int J Psychophysiol 47:65-74.

Corbetta M, Shulman GL (2002) Control of goal-directed and stimulusdriven attention in the brain. Nat Rev Neurosci 3:201-215.

Ergenoglu T, Demiralp T, Bayraktaroglu Z, Ergen M, Beydagi H, Uresin Y (2004) Alpha rhythm of the EEG modulates visual detection performance in humans. Brain Res Cogn Brain Res 20:376-383.

Fox MD, Corbetta M, Snyder AZ, Vincent JL, Raichle ME (2006) Spontaneous neuronal activity distinguishes human dorsal and ventral attention systems. Proc Natl Acad Sci USA 103:10046-10051.

Fu KM, Foxe JJ, Murray MM, Higgins BA, Javitt DC, Schroeder CE (2001) Attention-dependent suppression of distracter visual input can be crossmodally cued as indexed by anticipatory parieto-occipital alpha-band oscillations. Brain Res Cogn Brain Res 12:145-152.

Goldman RI, Stern JM, Engel Jr J, Cohen MS (2002) Simultaneous EEG and fMRI of the alpha rhythm. NeuroReport 13:2487-2492.

Gross J, Kujala J, Hamalainen M, Timmermann L, Schnitzler A, Salmelin R (2001) Dynamic imaging of coherent sources: studying neural interactions in the human brain. Proc Natl Acad Sci USA 98:694-699.

Gusnard DA, Raichle ME (2001) Searching for a baseline: functional imaging and the resting human brain. Nat Rev Neurosci 2:685-694.

Hämäläinen M, Hari R, Ilmoniemi RJ, Knuutila J, Lounasmaa OV (1993) Magnetoencephalography: theory, instrumentation, and applications to noninvasive studies of the working human brain. Rev Mod Phys 65:413-497.

Hari R, Salmelin R (1997) Human cortical oscillations: a neuromagnetic view through the skull. Trends Neurosci 20:44-49.

Huang M, Mosher JC (1997) A novel head model for the MEG forward problem: BEM accuracy with only spherical model complexity. NeuroImage 5:S441.

Hughes SW, Crunelli V (2005) Thalamic mechanisms of EEG alpha rhythms and their pathological implications. Neuroscientist 11:357-372.

Jensen O, Gelfand J, Kounios J, Lisman JE (2002) Oscillations in the alpha band $(9-12 \mathrm{~Hz})$ increase with memory load during retention in a shortterm memory task. Cereb Cortex 12:877-882.

Jensen O, Goel P, Kopell N, Pohja M, Hari R, Ermentrout B (2005) On the human sensorimotor-cortex rhythm: sources and modeling. NeuroImage 26:347-355.

Jokisch D, Jensen O (2007) Modulation of gamma and alpha activity during a working memory task engaging the dorsal or ventral stream. J Neurosci 27:3244-3251.

Jones SR, Pinto DJ, Kaper TJ, Kopell N (2000) Alpha-frequency rhythms desynchronize over long cortical distances: a modeling study. J Comput Neurosci 9:271-291.

Kelly SP, Lalor EC, Reilly RB, Foxe JJ (2006) Increases in alpha oscillatory power reflect an active retinotopic mechanism for distracter suppression during sustained visuospatial attention. J Neurophysiol 95:3844-3851.

Klimesch W, Sauseng P, Hanslmayr S (2007) EEG alpha oscillations: the inhibition-timing hypothesis. Brain Res Rev 53:63-88.

Klimesch W, Doppelmayr M, Rohm D, Pollhuber D, Stadler W (2000) Simultaneous desynchronization and synchronization of different alpha responses in the human electroencephalograph: a neglected paradox? Neurosci Lett 284:97-100.

Krause CM, Lang AH, Laine M, Kuusisto M, Porn B (1996) Event-related EEG desynchronization and synchronization during an auditory memory task. Electroencephalogr Clin Neurophysiol 98:319-326.

Laufs H, Kleinschmidt A, Beyerle A, Eger E, Salek-Haddadi A, Preibisch C, Krakow K (2003) EEG-correlated fMRI of human alpha activity. NeuroImage 19:1463-1476.

Liljeström M, Kujala J, Jensen O, Salmelin R (2005) Neuromagnetic localization of rhythmic activity in the human brain: a comparison of three methods. NeuroImage 25:734-745.

Linkenkaer-Hansen K, Nikulin VV, Palva S, Ilmoniemi RJ, Palva JM (2004) Prestimulus oscillations enhance psychophysical performance in humans. J Neurosci 24:10186-10190.

Lopes da Silva FH (1991) Neural mechanisms underlying brain waves: from neural membranes to networks. Electroencephalogr Clin Neurophysiol 79:81-93.

Maris E, Oostenveld R (2007) Nonparametric statistical testing of EEG- and MEG-data. J Neurosci Methods 164:177-190.

Medendorp WP, Kramer GF, Jensen O, Oostenveld R, Schoffelen JM, Fries P (2007) Oscillatory activity in human parietal and occipital cortex shows hemispheric lateralization and memory effects in a delayed double-step saccade task. Cereb Cortex 17:2364-2374.

Nichols TE, Holmes AP (2002) Nonparametric permutation tests for functional neuroimaging: a primer with examples. Hum Brain Mapp 15:1-25.

Palva S, Palva JM (2007) New vistas for alpha-frequency band oscillations. Trends Neurosci 30:150-158.

Percival DB, Walden AT (1993) Spectral analysis for physical applications: multitaper and conventional univariate techniques. Cambridge, UK: Cambridge UP.

Pfurtscheller G, Stancak Jr A, Neuper C (1996) Event-related synchronization (ERS) in the alpha band: an electrophysiological correlate of cortical idling [review]. Int J Psychophysiol 24:39-46.

Ray WJ, Cole HW (1985a) EEG activity during cognitive processing: influence of attentional factors. Int J Psychophysiol 3:43-48.

Ray WJ, Cole HW (1985b) EEG alpha activity reflects attentional demands, and beta activity reflects emotional and cognitive processes. Science 228:750-752.

Ress D, Backus BT, Heeger DJ (2000) Activity in primary visual cortex predicts performance in a visual detection task. Nat Neurosci 3:940-945.

Rougeul-Buser A, Buser P (1997) Rhythms in the alpha band in cats and their behavioural correlates. Int J Psychophysiol 26:191-203.

Salinas E, Thier P (2000) Gain modulation: a major computational principle of the central nervous system. Neuron 27:15-21.

Thut G, Nietzel A, Brandt SA, Pascual-Leone A (2006) Alpha-band electroencephalographic activity over occipital cortex indexes visuospatial attention bias and predicts visual target detection. J Neurosci 26:9494-9502.

Traub RD, Jefferys JG, Whittington MA (1999) Fast oscillations in cortical circuits, Ed 1. Cambridge, MA: MIT

Tuladhar AM, Huurne NT, Schoffelen JM, Maris E, Oostenveld R, Jensen O (2007) Parieto-occipital sources account for the increase in alpha activity with working memory load. Hum Brain Mapp 28:785-792.

Van Veen BD, van Drongelen W, Yuchtman M, Suzuki A (1997) Localization of brain electrical activity via linearly constrained minimum variance spatial filtering. IEEE Trans Biomed Eng 44:867-880.

Vanni S, Revonsuo A, Hari R (1997) Modulation of the parieto-occipital alpha rhythm during object detection. J Neurosci 17:7141-7147.

von Stein A, Chiang C, König P (2000) Top-down processing mediated by interareal synchronization. Proc Natl Acad Sci USA 97:14748-14753.

Worden MS, Foxe JJ, Wang N, Simpson GV (2000) Anticipatory biasing of visuospatial attention indexed by retinotopically specific alpha-band electroencephalography increases over occipital cortex. J Neurosci 20:RC63.

Yamagishi N, Goda N, Callan DE, Anderson SJ, Kawato M (2005) Attentional shifts towards an expected visual target alter the level of alpha-band oscillatory activity in the human calcarine cortex. Brain Res Cogn Brain Res 25:799-809. 